\title{
COPIM
}

\section{COPIM statement on the corporate acquisition of $O A$ infrastructure}

Community-led Open Publication Infrastructures for Monographs

Published on: Dec 15, 2021

License: Creative Commons Attribution 4.0 International License (CC-BY 4.0). 
At $\underline{\text { COPIM }}$, we have noted the recent acquisition of Knowledge Unlatched by Wiley, which itself follows Knowledge Unlatched's opaque transition in 2016 from a UK Community Interest Company (a non-profit organisation) into a German GmbH (roughly equivalent to a UK PLC, i.e. a for-profit company). This move by Wiley is one of several recent acquisitions of open access (OA) infrastructure by large commercial organisations, such as bepress by Elsevier in 2017, and F1000 Research by Taylor \& Francis in 2020. It reflects an ongoing consolidation of research infrastructure by major publishing corporations, and in particular the increasing attempts to monetise and, potentially, monopolise the infrastructures of open knowledge dissemination.

From its beginning, COPIM has been driven by the belief, held by all its partners ( $\underline{\mathrm{a}}$ consortium of universities, libraries, scholar-led OA publishers and research infrastructure providers) that the infrastructure we rely on to publish and disseminate OA books should itself be open, and owned and governed by the research communities that use it. We have repeatedly cited the widely-quoted argument by Bilder, Lin and Neylon that 'Everything we have gained by opening. content and data will be under threat if we allow the enclosure of scholarly_ infrastructures $^{\prime} \underline{1}$ and this motivates and shapes our work. The recent acquisitions of OA infrastructures by large for-profit corporations pose precisely this threat.

By contrast, the central philosophy of COPIM, which we have discussed publicly and written about extensively, is that of 'scaling small':

an alternative organisational principle for governing community-led publishing projects based on mutual reliance, care, and other forms of commoning [...] this principle eschews standard approaches to organisational growth that tend to flatten community diversity through economies of scale. Instead, it puts forward the idea that scale can be nurtured through intentional collaborations between community-driven projects that promote a bibliodiverse ecosystem while providing resilience through resource sharing and other kinds of collaboration. $\underline{2}$

Essentially, 'scaling small' describes an ecosystem of small, diverse publishers and publishing projects working in collaboration with other stakeholders in scholarly communication, such as universities, libraries and non-profit infrastructure providers, which is sustained and expanded by commonly-owned and governed infrastructure that enables each individual initiative to operate at its optimum level, whatever that may be. It envisions the flourishing and proliferation of smaller entities that do not need themselves to grow radically in size in order to do their work effectively, nor to be 
owned by a much larger organisation. A powerful expansion of OA book publishing could (and in our view should) therefore be achieved not by the growth and dominance of a small number of disproportionately large companies, but by facilitating OA book publishing among a greater number of presses operating at smaller scales. This would also better reflect the current landscape of academic book publishing, in which small and/or specialist presses make significant contributions to the publication of long-form academic research. $\underline{3}$ In other words, the 'scaling small' philosophy that COPIM is following is explicitly and intentionally an alternative to large-scale, commercial approaches to academic publishing.

\section{But how to put these values into practice?}

COPIM is building a number of models, systems and platforms as part of our work to remove hurdles preventing new and existing open access book initiatives from adopting open access workflows, specifically in the areas of funding, business models, dissemination, experimentation, and archiving. These include:

- The Open Book Collective (OBC), due to launch in spring 2022, a collective that brings together OA publishers, infrastructure providers, and libraries to facilitate easy assessment and support of community-led approaches to publishing, and to better integrate the research published by and shared via these initiatives;

- The OBC charter will establish that the collective and the revenue management platform that it supports cannot be taken over or incorporated by a for-profit entity or entities;

- Opening the Future, a model that enables closed-access presses to 'flip' to OA by offering closed-access backlist for subscription by libraries and using the revenue to fund OA frontlist;

- $\underline{\text { Thoth}}$, an open metadata management and dissemination system that will enable publishers to create high-quality, open metadata to share their OA books across multiple platforms and in a wide variety of formats.

It is central to our work that the infrastructure we build will be owned and governed by its communities of users in perpetuity. We will achieve this in two principal ways:

1. All the software we build is open source. All code is shared openly (currently available on GitHub ) for anyone to use. $\underline{5}$ This is a safeguard against the commercial capture of what we are building.

2. A crucial aspect of COPIM is our governance. Developing robust and long-lasting governance models for the infrastructure we build is a specific work package within 


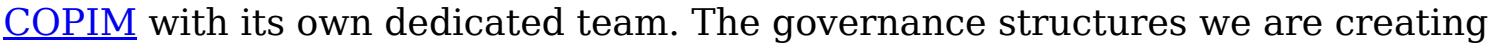
are co-designed together with the scholarly communications community to support a broad network of community-led scholarly communications organisations committed to the production, support, and long-term maintenance of a non-commercial open knowledge commons. We are creating inalienable systems that cannot be co-opted or overtaken by the interests of a few players or commercial entitities, and designing structures to ensure that we will remain community-driven. As with all our work, the development of our governance structures is being openly documented on our PubPub site. $\underline{6}$

We recognise that what we build will only succeed with the support of the communities we wish to serve, and of which we are part. But support requires trust, and trust requires appropriate levels of transparency and care. This post reflects our desire to tackle issues of governance and ownership carefully and transparently, and to make sure that openness and community governance are embedded structurally in what we do-particularly as we know libraries are becoming used to new initiatives with a good story appealing to them for funding, only later for the glossy corporate press release to drop. We welcome questions from anyone who is interested in any aspect of our work, including our governance: you can contact us at the addresses given below.

At COPIM we are part of a wider network of individuals and organisations developing or supporting open infrastructure, including $\underline{\text { SCOSS }}$, Invest in Open Infrastructure, the

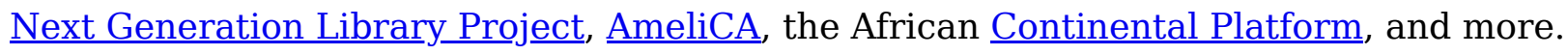
We add our voice to those arguing that infrastructure is not peripheral to academic research; on the contrary, it conditions and sustains it, and its creation, development and ongoing management is therefore a vital aspect of that research. We look forward to working with others to ensure that at least some of these essential structures will be unenclosed, open, and owned by the academic communities that rely on them.

If you are a library, publisher, or other organisation interested in working with us towards this goal, please contact us using the details below.

\section{Consortial Members of COPIM}

COPIM is an international partnership comprising

- universities: Coventry_University, Birkbeck, University_of London, Lancaster University, and Trinity_College,_Cambridge; 
- established scholar-led open access presses, represented through the $\underline{\text { ScholarLed }}$

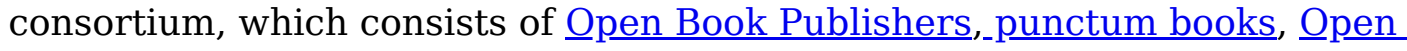

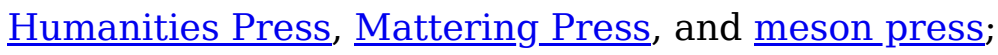

- libraries:University of California Santa Barbara (UCSB) Library, Loughborough University Library, the British Library;

- infrastructure providers: the Directory of Open Access Books (DOAB), Jisc;

- and membership organisations: the Digital Preservation Coalition.

\section{Contact COPIM}

- Email: $\underline{\text { info@copim.ac.uk }}$

- Twitter: @COPIMproject

- Website: https://www.copim.ac.uk/

- Open documentation site: https://copim.pubpub.org/

- Libraries and presses interested in learning more about COPIM's collaborative multistakeholder collective and platform, the Open Book Collective, please get in touch

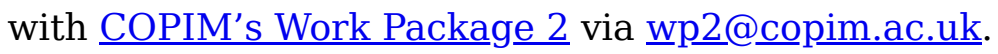

- If you are a library interested in subscribing to Opening the Future or a press interested in implementing it, or if you would like to know more about this particular model, visit https://openingthefuture.net/ or email OppeningTheFuture@copim.ac.uk.

- Presses interested in learning more about Thoth, COPIM's open metadata system,

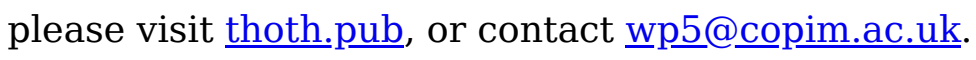

- And researchers, presses, and libraries interested in experimental approaches to long-form publishing in the Humanities and Social Sciences, please explore our recent report, visit copim.pubpub.org/work-package-6 or get in touch with wpㅡ@ㅡㅁim.ac.uk.

Header image by Omar Flores on Unsplash

\section{Footnotes}

1. Bilder G, Lin J, Neylon C (2015) Principles for Open Scholarly Infrastructure-v1, retrieved 9 Dec 2021, http://dx.doi.org/10.6084/m9.figshare.1314859. This has developed into the Principles of Open Scholarly Infrastructure (POSI), with which we are currently engaging as a project and considering how these apply to our work. See https://openscholarlyinfrastructure.org $L \doteq$

2. Abstract of Adema, J. \& Moore, S. A., (2021) "Scaling Small; Or How to Envision New Relationalities for Knowledge Production", Westminster Papers in Communication and Culture 16(1), p.27-45. doi: https://doi.org/10.16997/wpcc.918. 
See also https://copim.pubpub.org/search?q=scaling + small for more of the presentations and posts in which we have discussed this concept. $\bullet$

3. This argument is made by Barnes \& Gatti (2019), see: Lucy Barnes, Rupert Gatti. Bibliodiversity in Practice: Developing Community-Owned, Open Infrastructures to Unleash Open Access Publishing. ELPUB 2019 23rd edition of the International Conference on Electronic Publishing, Jun 2019, Marseille, France. 〈10.4000/proceedings.elpub.2019.21〉. 〈hal-02175276〉. They observe: 'Simon Tanner (2016) studies these book submissions to the REF2014 in greater detail, and highlights the remarkable range and diversity of publishers involved, many of which are small and/or specialist Presses. He notes that in total 1,180 unique publishers were associated with the books submitted to Panel D alone (Arts and Humanities), with the top 10 publishers accounting for less than $50 \%$ of submissions.' $ヒ$

4. While it is common practice to share open-source code via repositories such as GitHub, we note that GitHub itself is a commercial enterprise (owned by Microsoft) and as a project we are considering whether we ought to address this in some practical way, e.g. by mirroring repositories to GitLab.

5. See: Thoth, https://github.com/thoth-pub/; Opening the Future, https://github.com/BirkbeckCTP/otf-signup. The Open Book Collective platform is still in development and its code will be released soon. $\triangleq$

6. See for example Fathallah, J. (2021). Collective Governance: an Update from The Open Book Collective Work Package. COPIM.

https://doi.org/10.21428/785a6451.f47c5c51 and Moore, S. (2021). Exploring Models for Community Governance. COPIM. https://doi.org/10.21428/785a6451.0304a2a8 